\title{
A Numerical Investigation of Through-Tool Coolant Wetting Behaviour in Twist-Drilling
}

\author{
Adam Johns ${ }^{1}$, Eleanor Merson' ${ }^{2}$, Raphael Royer², Harvey Thompson1, Jonathan Summers ${ }^{1}$ \\ ${ }^{1}$ School of Mechanical Engineering, University of Leeds \\ Leeds, UK \\ a.johns@leeds.ac.uk; h.m.thompson@leeds.ac.uk; j.l.summers@leeds.ac.uk \\ ${ }^{2}$ Sandvik Coromant \\ Sheffield, UK \\ eleanor.merson@sandvik.com; raphael.royer@sandvik.com
}

\begin{abstract}
Internal coolant channels are a common method of transporting large thermal loads away from the tool in twist-drill machining to increase tool life and to aid chip evacuation and avoid catastrophic tool failure. A finite element model, loosely coupled with a multiphase Computational Fluid Dynamics (CFD) model is used to investigate the distribution of coolant in the bore hole and the effect of channel position on cutting geometry lubrication. The use of response surface models show that all designs do not fully flood the bore hole and that not all areas of the tool geometry are lubricated with coolant. Visual analysis of CFD results show that coolant, for all designs, primarily lubricates the area between the cutting edge and the coolant hole exit, however depending on application requirements coolant channel positioning can be used to modify coolant supply to the axial rake, for chip evacuation or to the cutting edge for heat removal.
\end{abstract}

Keywords: Drilling Coolant CFD OpenFOAM VOF

\section{Introduction}

Twist-drill machining is a process of creating cylindrical holes frequently used in the manufacture of cars, trains, planes and ships and are typically comprised of two components, the drill and the cutting tool (referred to as the drill bit). The drill is the mechanism responsible for rotating and supplying forces to the drill bit, while the drill bit applies the forces to cut the workpiece. The improvement of drill bit performance is of particular interest to the manufacturing industry in order to reduce component costs and improve component quality. However, one of the central challenges of increasing productivity is the management of large thermomechanical loads. Since the first report of cutting fluids in 1894 by F. Taylor, liquid coolants have been increasingly used to handle these loads and extend tool life [1]. One common method of coolant application is via internal coolant channels, which are manufactured into the flute of the tool, illustrated in Figure 1, to supply liquid emulsion coolant directly to the cutting geometry to transport thermal energy away from the tool [2] and aid chip evacuation [3].

The experimental observation of coolant application is particularly challenging due to the combination of small enclosed bore hole geometry and large tool angular velocities. As a result, it is difficult to improve the usage of coolant with little knowledge of the underlying delivery mechanisms. In light of these limitations studies are typically limited to post-experiment tool inspection [5, 6] or through cutting force telemetry data analysis. These experimental challenges have lead to numerous research papers adopting Computational Fluid Dynamics (CFD) to numerically study coolant performance.

CFD has been employed to investigate a number of different drilling applications such as internal coolant channels in oil-well drilling [7], the tool wear in the oil and gas industry [8] and MQL applications [3]. In twist-drill machining, research by Obikawa et al. [9] studied coolant flow between the tool flank face and the workpiece surface in response to changes in flow rate. This work found that the narrow space between the flank face and workpiece limited the supply of coolant and an increase in flow rate improved supply of coolant to the cutting geometry.

Beer et al. [6] used a CFD model, validated by tool wear inspections, to investigate the effects of grooves in the flank face on tool life and hole quality in the drilling of inconel 718. The addition of grooves in the flank face was found to increase tool life by up to 50\%. A recent study by Fallenstein et al. [10] used CFD to study the single phase flow of coolant within the bore hole and predict the amount of heat removed by coolant. Both channel positioning and flow rate were found to have a significant impact on the amount of heat removed from the tool. The benefit of increased flow rate was consistent across all designs and channel circumferential position had a greater effect on heat transfer over the radial positioning.

Oezkaya et al. [5] also employed a single-phase model to study the effects of coolant hole position and flow rate on tool-coolant 

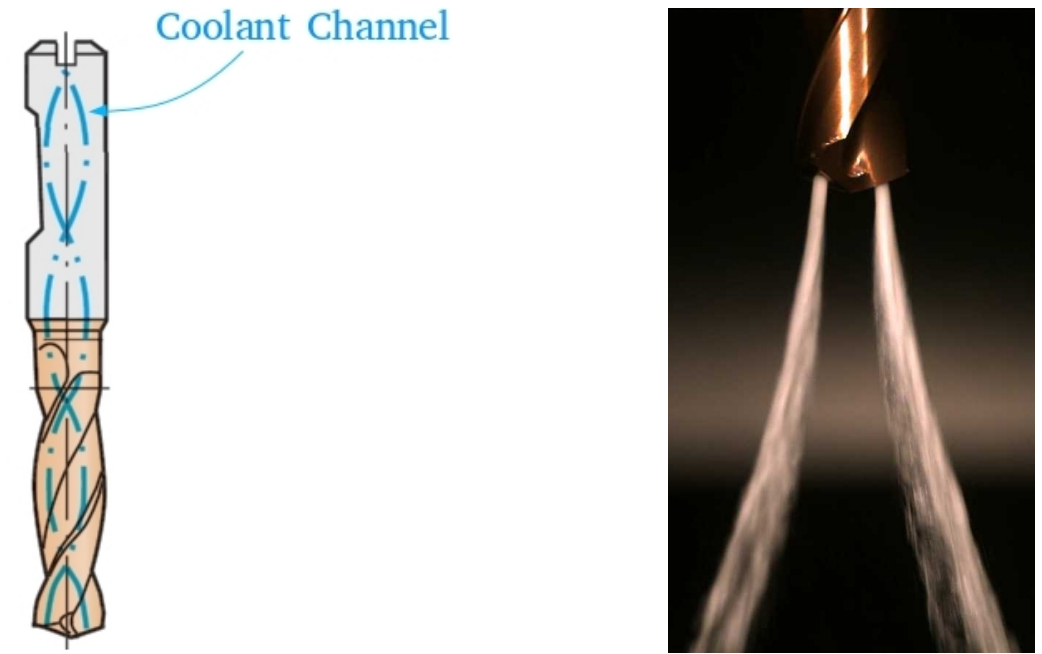

Fig. 1: Schematic diagram of a conventional twist-drill [4].

heat transfer in inconel 718 drilling. It was found that coolant is primarily distributed into the flute and identified 'dead' zones by the cutting edge corner. Increasing the supply pressure was found to have the greatest impact on tool cooling as well as increasing the coolant supply to these stagnant zones.

This work contributes to the existing literature on coolant flow by studying the distribution behaviour which has not previously been documented and would not otherwise be observable using single phase modeling formulations. Issues with swept meshing approaches are addressed using a Finite Element (FE) model to calculate the bore hole geometry profile and chip formation which is later used in the CFD model spatial discretisation. In this work a Volume of Fluid (VOF) CFD model tracks the distribution of coolant inside a realistic bore hole geometry combined with response surface models to analyse channel positioning effects on domain filling behaviour and the percentage lubricated area of the tool. Finally, visual analysis of numerical tool surface coolant distributions is used to study tool component wetting in response to changes in channel positioning.

\section{Model Formulation}

The numerical model outlined in this article is constructed from two loosely coupled numerical models:

The first is a FE model of the machining process to simulate the cutting process and is responsible for calculating the formation of the workpiece and chip. These simulations are carried out using ThirdWave Systems AdvantEdge. Further information regarding simulation set up is provided in Section 3.

The second model, referred to as the fluid model, is a Finite Volume (FV) model implemented in OpenFOAM [11] and uses the workpiece geometry calculated in the FE model to create a computational mesh accounting for the tool, chip and work-piece geometry. The fluid model is a two-phase model using the VOF method to track the coolant/air interface about a realistic tool-chip geometry. Further details are given in Section 4.

\section{Machining Model}

This paper uses ThirdWave Systems AdvantEdge version 7.1 simulation package to calculate the workpiece formation. The software, presented originally in [12], is used to simulate solid carbide drilling of steel. The software is based on a dynamic explicit Lagrangian FE model which employs adaptive remeshing to minimise element distortion. The tool used for this model is a 8mm diameter Sandvik Coromant CoroDrill 460 and the workpiece material is AISI-4140. The material model used for the workpiece and tool are part of the standard material library included in the software. Simulation parameters used a workpiece starting depth of 8mm with initial temperature 20 degrees Celsius. The simulation calculated 3 complete tool rotations to ensure a large continuous chip had formed using a spindle speed of 3970 RPM and $0.2 \mathrm{~mm}$ feed per revolution. Dynamic mesh refinement is used locally around the tool cutting edge to ensure adequate mesh resolution to resolve the cutting process; no mesh coarsening was authorised in the simulation in order to maintain the morphology of the simulated chip. The initial workpiece mesh consists of 138,053 tetrahedral elements, and the number of elements increases as the cutting progresses due to the adaptive remeshing. A total of 2139117 time steps were needed to complete the simulation as well as 1369 mesh refinement steps. Eight Central Processing Units (CPU) were used to carry out the simulation with a total CPU time of $292 \mathrm{~h} 32 \mathrm{~min}$. 


\section{Fluid Model}

The present work considers water-miscible coolant which is approximately $95 \%$ water and is therefore modelled as water. Both the air and coolant phases are treated as Newtonian and incompressible and the rate of strain tensor is linearly related to the stress tensor. A similar approach to the VOF method described in [13] is used to track the large quantities of coolant [14], as implemented in the OpenFOAM CFD library [11]. The formulation of the model is given below:

$$
\begin{gathered}
\nabla \cdot \mathbf{U}=0 \\
\frac{\partial \alpha}{\partial t}+\nabla \cdot(\mathbf{U} \alpha)+\nabla \cdot\left[\mathbf{U}_{r} \alpha(1-\alpha)\right]=0 \\
\frac{\partial \rho \mathbf{U}}{\partial t}+\nabla \cdot(\rho \mathbf{U} \mathbf{U})-\nabla \cdot(\mu \nabla \mathbf{U})-(\nabla \mathbf{U}) \cdot \nabla \mu=-\nabla p_{d}-\mathbf{g} \cdot \mathbf{x} \nabla \rho+\sigma \kappa \nabla \alpha
\end{gathered}
$$

where $\mathbf{U}$ is velocity and $\mathbf{U}_{r}$ is the relative velocity defined as $\mathbf{U}_{r}=\mathbf{U}_{l}-\mathbf{U}_{g}$, where subscript $l$ and $g$ correspond to the liquid and gas velocities respectively. $\alpha$ is the volume fraction and $\mu$ and $\rho$ are the volume averaged viscosity and density respectively.

In order to simplify the definition of boundary conditions pressure is modified so that a single pressure system is considered where the normal component of the pressure gradient at a stationary non-vertical no-slip wall must be different for each phase due to the hydrostatic component $\rho \mathbf{g}$ when the phases are separated at the wall, i.e. if a contact line exists. In order to simplify the definition of boundary conditions it is common to define a modified pressure. Here pressure is modified by the density gradient and the body force due to gravity given by $p_{d}=p-\rho \mathbf{g} \cdot \mathbf{x}$. Where $\mathbf{x}$ is the position vector and $\mathbf{g}$ acceleration due to gravity

Surface tension is accounted for within the momentum equation by including it as a body force, $f_{s v}$. Surface tension at the liquid gas interface generates an additional pressure gradient, which is evaluated per unit volume using the continuum surface model formulation of Brackbill et al. [15]:

$$
\mathbf{f}_{s v}=\sigma \kappa \hat{\mathbf{n}} \delta_{s}
$$

Where $\sigma$ is the coefficient of surface tension of $0.07 \mathrm{kgs}^{-2}, \hat{\mathbf{n}}$ is the normal vector of the interface, $\delta_{s}$ is the interface delta function and $\kappa$ is the mean curvature of the interface defined as:

$$
\kappa=-\nabla \cdot\left(\frac{\nabla \alpha}{|\nabla \alpha|}\right)
$$

The presented VOF formulation has been evaluated against a range of multiphase flow problems, such as modulated jets [16], droplet impact and crater formation [17], film falling over turbulence wires [18] and inertia dominated and surface tension dominated flow regimes [19].

By weighing coolant captured in a container across a fixed time period the average fluid velocity exiting the tool was calculated at $80 \mathrm{~ms}^{-1}$. From this the Reynolds number was calculated at approximately 79000 indicating that the flow is highly turbulent and is accounted for using the realisable $k-\varepsilon$ turbulence model. The boundary conditions used specify the velocity at the inlet as $80 \mathrm{~ms}{ }^{-1}$ and pressure zero gradient, at the outlet atmospheric pressure is specified and an outlet condition is selected for velocity. For the tool and workpiece walls zero velocity no slip conditions are specified and for pressure zero gradient. The effects of rotation were not included in this simulation as the inertia of the liquid phase dominates the Coriolis forces, following the findings of [20].

\section{Computational Geometry}

The computational mesh for the fluid domain, the cavity inside the borehole encompassing the workpiece and tool, is created with snappyHexMesh using the tool CAD geometry and the workpiece geometry calculated in the FEM model as inputs. The resulting mesh of the fluid domain includes the chip, the shape of the borehole bottom and the clearance between the tool margin and the workpiece wall. As a result of modelling the clearance between the margin and the workpiece, each coolant channel is no longer isolated from the other and both coolant channels are modelled using this approach. Surface based refinement was used about the workpiece and tool surfaces and the resulting meshes were constructed from roughly 2 million cells each with a maximum cell volume of $8.38 \times 10^{-13} \mathrm{~m}^{3}$ and minimum of $8.53 \times 10^{-15} \mathrm{~m}^{3}$. Grid sensitivity was checked by comparing the results against another with 5 million control volumes and was found to be sufficient as the relative variation between the results was $1 \%$.

\subsection{Validation - Experimental}

The CFD model is validated experimentally using a sacrificial polymer coating to indicate coolant delivery regions. This analysis was performed by first coating the tool in a proprietary polymer made from Nitrocellulose and coloured to aid visual inspection, then inserting the tool into a pre-drilled blind hole of depth three times tool diameter $0.4 \mathrm{~mm}$ from the bore hole bottom. Coolant is supplied for 20 seconds before removing the tool and visually inspecting for surface regions where the polymer coating has been removed by the flow of coolant. The surface regions where the polymer coating has been removed indicates where coolant is supplied after exiting the tool.

Experimental results are presented in Figure 3(a) and show that the polymer coating has mostly been removed on the flank face between the coolant channel and the cutting edge and across the axial rake. Figure 3(a) shows the distribution of coolant predicted using 


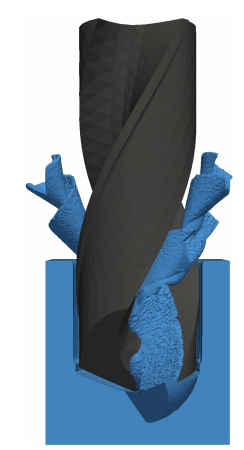

Fig. 2: Geometry obtained using Thirdwave Systems AdvantEdge showing the workpiece in blue and the tool in grey.

CFD across the tool surface where the blue areas indicate zones supplied with coolant and the red areas indicate regions which are not supplied with coolant. The profile of coolant calculated by the CFD model is clearly in qualitatively agreement with the profile shown in the experiment. Furthermore, the CFD model is also predicting that coolant is not directly supplied to the tertiary clearance or the tool flute, which is also reflected in the experimental results.

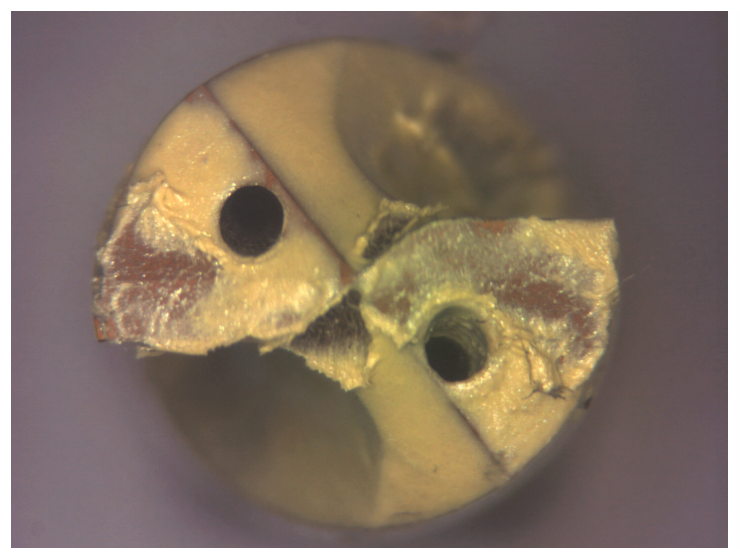

(a) Experimental

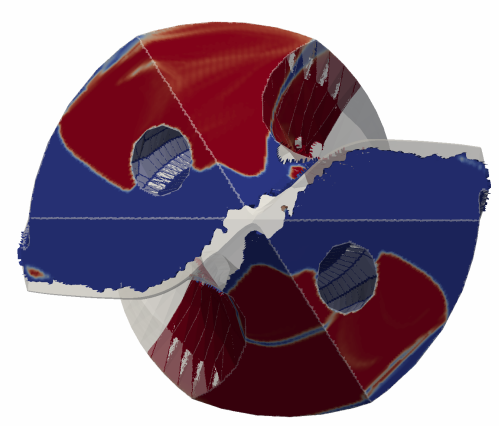

(b) CFD

Fig. 3: Comparison of experimental and CFD results..

\section{Coolant Channel Analysis}

In this paper, the effects of coolant channel exit position on domain filling and tool wetting behaviour will be studied. Each coolant channel is specified relative to its radial positioning $r$ and circumferential position $\theta$ illustrated in Figure 4 . In the following analysis sections, each design evaluated has been analysed for its domain flooding behaviour and its tool wetting response. Domain flooding analysis calculates the percentage of the bore hole flooded by coolant, or how long it takes for the cutting zone to become fully flooded. Both of which will influence drill cooling and is calculated by:

$$
\sum_{i=1}^{N} V_{i} \alpha_{i}
$$

where $N$ is the number of control volumes, $V_{i}$ is the volume of control volume $i$ and $\alpha_{i}$ is the volume fraction of control volume $i$. To aid analysis this represented as a percentage of the total domain volume. 


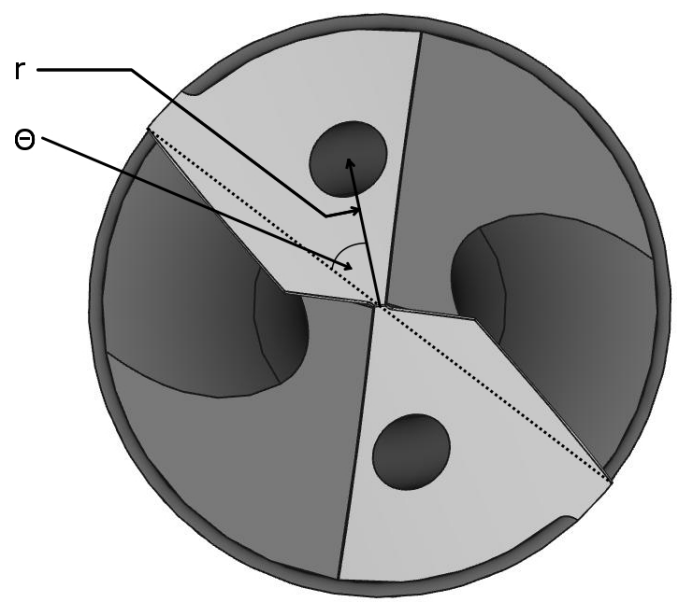

Fig. 4: Channel position parameters.

Tool wetting calculates the area of the tool coated in coolant. This is calculated by:

$$
\sum_{i=1}^{N_{f}} A_{f_{i}} \alpha_{f_{i}}
$$

where $N_{f}$ is the number of faces used to represent the tool surface, $A_{f_{i}}$ is the area of face $i$ and $\alpha_{f_{i}}$ is the volume fraction as face $i$. Tool wetting is represented in the later sections as a percentage of the total surface area.

\section{Response Surface Modelling}

The effect of coolant channel exit circumferential positioning, $\theta$, and radial positioning, $r$, on tool wetting and bore hole flooding is shown through response surfaces. The response surfaces are built using a Moving Least Squares (MLS) metamodel from steady state CFD solutions of designs obtained from an Optimum Latin Hypercube Design of Experiments (DoE) of 20 points in the domain where $2 \mathrm{~mm} \leq r \leq 6 \mathrm{~mm}$ and $12^{\circ} \leq \theta \leq 72^{\circ}$, see e.g. [21]. The DoE points and the calculated flooding and tool wetting are given in Table 1. The MLS metamodel uses a Gaussian weight decay function to determine the weighting of points in the regression analysis at each design point $\underline{x}_{i}=\left(r_{i}, \theta_{i}\right)$ :

$$
w_{i}(\underline{x})=\exp \left(-\beta\left\|\underline{x}-\underline{x_{i}}\right\|^{2}\right)
$$

where $\left\|\underline{x}-x_{i}\right\|=\left(r-r_{i}\right)^{2}+\left(\theta-\theta_{i}\right)^{2}$ is the Euclidean distance between the design point $\underline{x}=(r, \theta)$ at which the MLS metamodel is being evaluated, and the $i$ th DoE point $\left(r_{i}, \theta_{i}\right)$, and $\beta$ is the closeness of fit parameter. A large value of $\beta$ ensures the MLS metamodel reproduces the known DoE point values accurately, whereas a smaller value of $\beta$ can reproduce the effect of numerical noise on the metamodel [22]. The vales of $\beta$ for the metamodel are selected by minimising the Root Mean Square Error (RMSE) between the predictions from the metamodel and those from the CFD at the DoE points. Following [23] Leave One Out (LOO) cross validation is used, where a DoE point is removed from the metamodel and a metamodel is created using the remaining 19 points. The removed point is used to evaluated the model RMSE. This is repeated for every DoE point and the average RMSE across the set of 20 DoE points is calculated.

The response surface calculated for domain flooding is given in Figure 5(a) with an optimum $\beta=19$ and RMSE of 0.9. The resulting surface shows that no designs fully flood the domain and that the designs which fill the cavity have a greater circumferential positioning and radial spacing. The designs which fill the fluid domain the least have the smallest radial distance and are positioned closer to the cutting edge. This response surface also shows that $r$ appears to have little effect unless $\theta$ is very large.

A metamodel showing the percentage of the tool surface coated in coolant as a function of $r$ and $\theta$ is given in Figure 5(b). The metamodel was created using $\beta=31$ and a resulting RMSE of 50.3. The results show that there is a complex relationship between $r$ and $\theta$ with several locally optimal designs. Interestingly, these show that no design lubricates the entire tool and that there must be components of the external geometry not supplied with coolant. The design with global maximum surface wetting was found using a particle swarm genetic algorithm, which is located at $r=6$ and $\theta=56.06$. This, combined with the flooding response surface show that designs with large $r$ and $\theta$ tend to flood more of the bore hole and lubricate a greater area of the tool. 
Table 1: Design of Experiments and CFD results.

\begin{tabular}{|c|c|c|c|c|}
\hline Design \# & $r$ & $\theta$ & \% Flooded & \% Wetted \\
\hline 1 & 2 & 27.7096 & 13 & 38 \\
\hline 2 & 2.21052 & 65.6044 & 22 & 48 \\
\hline 3 & 2.42104 & 52.9726 & 19 & 44 \\
\hline 4 & 2.63156 & 37.183 & 17 & 40 \\
\hline 5 & 2.84212 & 18.2356 & 15 & 35 \\
\hline 6 & 3.05264 & 62.4466 & 22 & 48 \\
\hline 7 & 3.26316 & 49.8148 & 20 & 43 \\
\hline 8 & 3.47368 & 30.8674 & 18 & 38 \\
\hline 9 & 3.6842 & 15.0778 & 17 & 43 \\
\hline 10 & 3.89472 & 71.92 & 24 & 51 \\
\hline 11 & 4.10528 & 40.3408 & 21 & 44 \\
\hline 12 & 4.3158 & 59.2882 & 23 & 50 \\
\hline 13 & 4.52632 & 24.5518 & 19 & 43 \\
\hline 14 & 4.73684 & 46.657 & 23 & 53 \\
\hline 15 & 4.94736 & 11.92 & 17 & 40 \\
\hline 16 & 5.15788 & 68.7622 & 25 & 51 \\
\hline 17 & 5.36844 & 34.0252 & 21 & 42 \\
\hline 18 & 5.57896 & 56.1304 & 26 & 55 \\
\hline 19 & 5.78948 & 21.3934 & 19 & 39 \\
\hline 20 & 6 & 43.4992 & 28 & 53 \\
\hline \multicolumn{5}{|c}{}
\end{tabular}

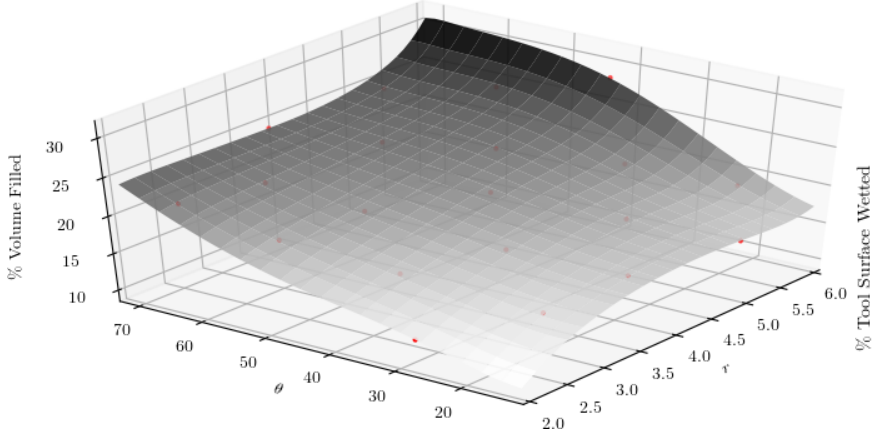

(a) Flooding

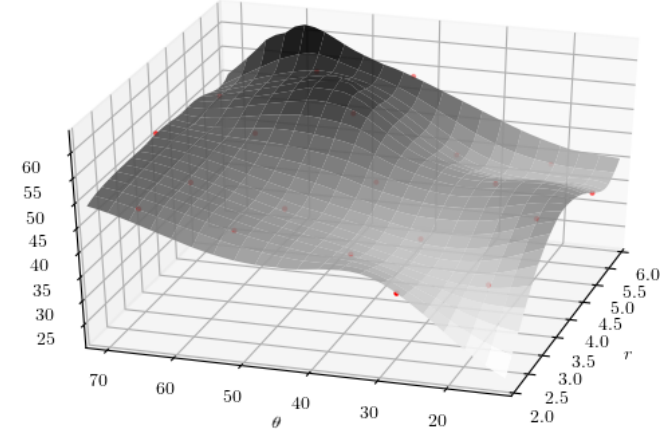

(b) Wetting

Fig. 5: Moving least squares response surfaces for bore hole flooding and tool wetting.

\section{Wetting Profiles}

The previously discussed metamodels are effective at giving a simple overview of the effects of radial and circumferential positioning of coolant channels. However, measuring the response of the system in terms of a percentage of the tool covered does not indicate which components of the external geometry are supplied with coolant. Figure 6 qualitatively analyses the distribution of coolant about the tool surface by colouring the tool geometry of each DoE point by the steady state distribution of coolant. In order to aid the visualisation the workpiece and chip have been subtracted from the image.

The geometries examined are displayed in Figure 6. In this figure the tool surfaces are coloured by the volume fraction at the simulation steady state, where the blue areas indicate wetted areas $(\alpha=1)$ and red areas indicate areas of the surface which are not $(\alpha=0)$. It can clearly be seen that all designs follow the profile of providing coolant directly to the back of the cutting edge and either into the workpiece walls or into the axial rake. However the amount of the flank face which is supplied coolant is sensitive to the location of the coolant channel exit position as it appears to primarily coat the area between the coolant hole and the cutting edge. From this collection of results it can be seen that not a significant amount of coolant is directed up the flute of the tool, which would otherwise be evidenced by more coolant up the secondary clearance or on the flute face. The amount of coolant directed across the axial rake appears 
to be influenced by the radial distance of the coolant channel more significantly than the circumferential positioning as designs with larger radial positioning are shown to provide more coolant to the axial rake of the tool than designs with smaller $r$. This suggests that tool designs which favour chip evacuation using coolant may benefit from positioning channels with large circumferential and radial positioning in order to increase flow to the axial rake, whereas tools which require increase heat transfer would benefit from minimising circumferential position to focus the flow around the cutting edge.
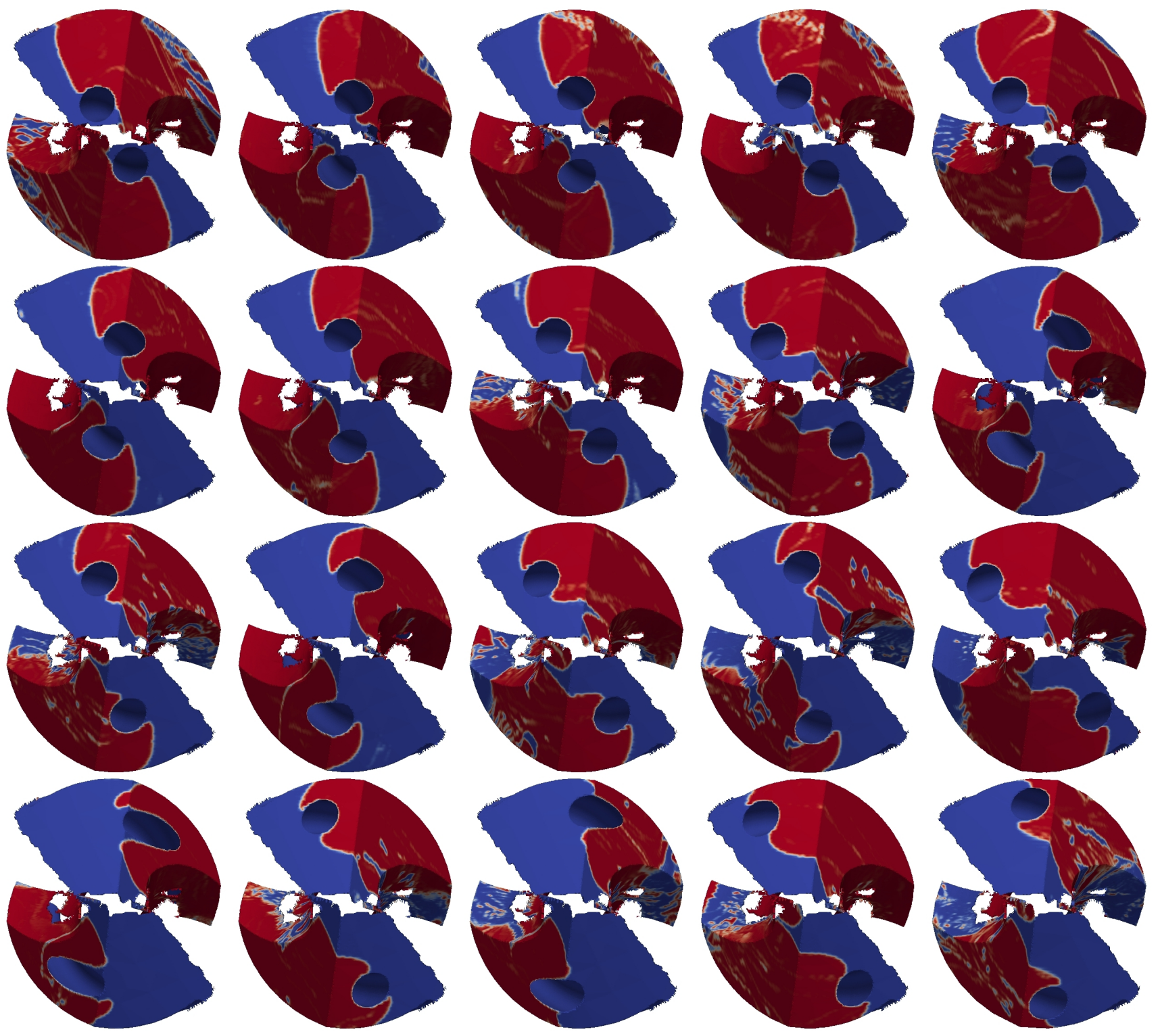

Fig. 6: Tool tip geometries with different coolant channel exit position coloured by coolant volume fraction. Blue indicates areas where coolant is supplied and red where coolant is neglected.

\section{Conclusions}

Previous research has shown coolant improves drill bit performance by lubricating the cutting zone, removing heat and aiding chip evacuation. The central motivation of this work is to understand how these improvements are achieved through studying how coolant is distributed inside the bore hole. Previous CFD modelling of twist-drill coolant employ single phase formulations to predict trajectory and heat transfer. This work is the first to employ a multiphase CFD model to study the distribution and wetting profile in twist-drill machining. This is combined with a FE model to calculate a realistic bore hole geometry for the CFD meshing process. 
Response surface modelling of domain flooding show that the position of the coolant channel does not significantly effect the cavity filling and no design fully floods the domain. The designs which maximise the domain flooding achieve approximately $30 \%$ flooding and maximise both circumferential and radial spacing. Wetting response surfaces found that no designs fully lubricated the tool, but tools with greater circumferential positioning cover a greater percentage area of the tool than channels with smaller circumferential positioning. Coolant channels which are located further from the centre of the tool also increased the percentage area of the tool coated in coolant, however had a smaller effect than circumferential positioning.

Profile wetting analysis was used to evaluate tool surface coolant distribution for each channel configuration to identify the geometry components which are supplied coolant. All of the tested designs displayed a similar coolant distribution pattern where coolant is supplied along the tool flank face between the coolant holes and the cutting edge before being directed into the workpiece walls or across the tool axial rake. The results show that as the circumferential distance increases, a larger area of the flank face is coated in coolant. Small circumferential positioned coolant channels, channels which are closer to the cutting edge, appear to coat less of the tool with coolant but highly localise the wetting about the cutting edge, the primary source of heat. Finally, visual analysis of the CFD results showed that the quantity of coolant directed either to the tool margin or across the axial rake can be controlled through coolant channel positioning, channels with large $r$ and $\theta$ direct more coolant across the axial rake, while tools with small $r$ direct less coolant across the axial rake.

\section{References}

[1] R. Avila and A. Abrao, "The effect of cutting fluids on the machining of hardened aisi 4340 steel," Journal of Materials Processing Technology, vol. 119, no. 1, pp. 21-26, 2001.

[2] D. Haan, S. Batzer, W. Olson, and J. Sutherland, "An experimental study of cutting fluid effects in drilling," Journal of Materials Processing Technology, vol. 71, no. 2, pp. 305-313, 1997.

[3] D. U. Braga, A. E. Diniz, G. Miranda, and N. Coppini, "Using a minimum quantity of lubricant (mql) and a diamond coated tool in the drilling of aluminum-silicon alloys," Journal of Materials Processing Technology, vol. 122, no. 1, pp. 127-138, 2002.

[4] P. Zhang, N. Churi, Z. J. Pei, and C. Treadwell, "Mechanical drilling processes for titanium alloys: a literature review," Machining Science and Technology, vol. 12, no. 4, pp. 417-444, 2008.

[5] E. Oezkaya, N. Beer, and D. Biermann, "Experimental studies and cfd simulation of the internal cooling conditions when drilling inconel 718," International Journal of Machine Tools and Manufacture, vol. 108, pp. 52-65, 2016.

[6] N. Beer, E. Özkaya, and D. Biermann, "Drilling of inconel 718 with geometry-modified twist drills," Procedia CIRP, vol. 24, pp. 49-55, 2014.

[7] F. A. R. Pereira, M. A. S. Barrozo, and C. H. Ataíde, "Cfd predictions of drilling fluid velocity and pressure profiles in laminar helical flow," Brazilian Journal of Chemical Engineering, vol. 24, pp. 587 - 595, 122007.

[8] G. Watson, N. Barton, G. Hargrave et al., "Using new computational fluid dynamics techniques to improve pde bit performance," in SPE/IADC Drilling Conference. Society of Petroleum Engineers, 1997.

[9] T. Obikawa and M. Yamaguchi, "Computational fluid dynamic analysis of coolant flow in turning," Procedia CIRP, vol. 8, pp. 271-275, 2013.

[10] F. Fallenstein and J. Aurich, "Cfd based investigation on internal cooling of twist drills," Procedia CIRP, vol. 14, no. 0, pp. 293 298, 2014, 6th CIRP International Conference on High Performance Cutting, HPC2014.

[11] H. G. Weller, G. Tabor, H. Jasak, and C. Fureby, "A tensorial approach to computational continuum mechanics using object-oriented techniques," Computers in physics, vol. 12, no. 6, pp. 620-631, 1998.

[12] T. Marusich and M. Ortiz, "Modelling and simulation of high-speed machining," International Journal for Numerical Methods in Engineering, vol. 38, no. 21, pp. 3675-3694, 1995.

[13] C. W. Hirt and B. D. Nichols, "Volume of fluid (vof) method for the dynamics of free boundaries," Journal of computational physics, vol. 39, no. 1, pp. 201-225, 1981.

[14] O. Ubbink, "Numerical prediction of two fluid systems with sharp interfaces," Ph.D. dissertation, University of London UK, 1997.

[15] J. Brackbill, D. B. Kothe, and C. Zemach, "A continuum method for modeling surface tension," Journal of computational physics, vol. 100, no. 2, pp. 335-354, 1992.

[16] V. Srinivasan, A. J. Salazar, and K. Saito, "Modeling the disintegration of modulated liquid jets using volume-of-fluid (vof) methodology," Applied Mathematical Modelling, vol. 35, no. 8, pp. 3710-3730, 2011.

[17] E. Berberović, N. P. van Hinsberg, S. Jakirlić, I. V. Roisman, and C. Tropea, "Drop impact onto a liquid layer of finite thickness: Dynamics of the cavity evolution," Physical Review E, vol. 79, no. 3, p. 036306, 2009.

[18] H. Raach, S. Somasundaram, and J. Mitrovic, "Optimisation of turbulence wire spacing in falling films performed with openfoam," Desalination, vol. 267, no. 1, pp. 118-119, 2011.

[19] S. S. Deshpande, L. Anumolu, and M. F. Trujillo, "Evaluating the performance of the two-phase flow solver interfoam," Computational Science \& Discovery, vol. 5, no. 1, p. 014016, 2012.

[20] A. S. Johns, "Computational fluid dynamic modelling and optimisation of internal twist-drill coolant channel flow," Ph.D. dissertation, University of Leeds, 2015.

[21] A. Al-Damook, N. Kapur, J. Summers, and H. Thompson, "Computational design and optimisation of pin fin heat sinks with rectangular perforations," Applied Thermal Engineering, 2016.

[22] C. Gilkeson, V. Toropov, H. Thompson, M. Wilson, N. Foxley, and P. Gaskell, "Dealing with numerical noise in cfd-based design optimization," Computers \& Fluids, vol. 94, pp. 84-97, 2014.

[23] G. de Boer, L. Gao, R. Hewson, H. Thompson, N. Raske, and V. Toropov, "A multiscale method for optimising surface topography in elastohydrodynamic lubrication (ehl) using metamodels," Structural and Multidisciplinary Optimization, pp. 1-15, 2016. 